Check for updates

Cite this: Phys. Chem. Chem. Phys., 2017, 19, 12136

Received 14th February 2017 Accepted 10th April 2017

DOI: $10.1039 / c 7 c p 00986 k$

rsc.li/pccp

\title{
Determination of the diffusion coefficient of hydrogen ion in hydrogels $\dagger$
}

\author{
Gábor Schuszter, $\ddagger^{a}$ Tünde Gehér-Herczegh, $\ddagger^{a}$ Árpád Szűcs, ${ }^{a}$ Ágota Tóth ${ }^{a}$ and \\ Dezső Horváth (1D*b
}

\begin{abstract}
The role of diffusion in chemical pattern formation has been widely studied due to the great diversity of patterns emerging in reaction-diffusion systems, particularly in $\mathrm{H}^{+}$-autocatalytic reactions where hydrogels are applied to avoid convection. A custom-made conductometric cell is designed to measure the effective diffusion coefficient of a pair of strong electrolytes containing sodium ions or hydrogen ions with a common anion. This together with the individual diffusion coefficient for sodium ions, obtained from PFGSE-NMR spectroscopy, allows the determination of the diffusion coefficient of hydrogen ions in hydrogels. Numerical calculations are also performed to study the behavior of a diffusion-migration model describing ionic diffusion in our system. The method we present for one particular case may be extended for various hydrogels and diffusing ions (such as hydroxide) which are relevant e.g. for the development of $\mathrm{pH}$-regulated self-healing mechanisms and hydrogels used for drug delivery.
\end{abstract}

\section{Introduction}

Although diffusion is usually considered to be slow as a transport mechanism, it plays an important role in many applications, especially when the length scale of the problem is microscopic or the time scale is sufficiently long. In these cases, the difference in the diffusion properties of chemical species may significantly affect the output. This effect of varying diffusion may be represented via reaction-diffusion driven pattern formation, where visible patterns facilitate the understanding of the underlying interactions.

Such chemical systems have been thoroughly investigated in the past few decades by both numerical studies and experimental studies. Notably in the early 1990s, stationary Turing patterns ${ }^{1}$ were first observed and since then well-designed experimental methods ${ }^{2,3}$ have been developed to produce these phenomena in various systems. Numerical simulations have shown that a rich variety of complex spatiotemporal patterns can be created using simple models with autocatalytic chemical reactions influenced by diffusion. ${ }^{4}$ The resulting patterns may be e.g. spirals, stripes, hexagons, lamellae, and self-replicating spots which have also been reproduced experimentally in acid auto-activated reactions in hydrogel media. ${ }^{5}$ Such intriguing

\footnotetext{
${ }^{a}$ Department of Physical Chemistry and Materials Science, Rerrich Béla ter 1., 6720 Szeged, Hungary

${ }^{b}$ Department of Applied and Environmental Chemistry, Rerrich Béla ter 1., 6720 Szeged, Hungary. E-mail: horvathd@chem.u-szeged.hu; Fax: +36 62/546-482; Tel: +36 62/544-614

$\dagger$ Electronic supplementary information (ESI) available. See DOI: 10.1039/ c7cp00986k

\# These authors contributed equally to the work.
}

acid auto-activated systems including, among others, the thioureaiodate-sulfite reaction which exhibited Turing bifurcation, the clock reaction bromate-sulfite system, ${ }^{6}$ the mixed Landolt-type pH-oscillator bromate-sulfite-ferrocyanide reaction, ${ }^{7}$ and other halogen-free hydrogen-peroxide driven reactions ${ }^{8}$ are all examples where phenomena like stationary patterns, spatial bistability, travelling acid-base fronts or spatiotemporal oscillations have been observed. Propagating fronts have been studied in e.g. the iodate-arsenous acid $^{9}$ or the chlorite-tetrathionate reactions as well. ${ }^{10,11}$ In other systems, the diffusion of hydroxide ions plays an important role regarding the macroscopic patterns. ${ }^{12} \mathrm{~A}$ common feature of these systems is that the pattern formation is essentially controlled by the diffusion rate of the chemical species present in the system, particularly regarding the autocatalyst that is often the hydrogen ion. Its fast hopping mechanism in water results in a high diffusion coefficient value and a longrange activation process that can significantly influence the nonlinear behavior of the system - e.g. oscillatory states - as shown by numerical studies. ${ }^{13}$

Hydrogels such as agarose, gelatin or polyacrylamide are fundamentally used as reaction media for chemical pattern formation for the purpose of eliminating macroscopic convection. In addition, $\mathrm{pH}$-sensitive hydrogels are also applied to make artificial molecular motors which are able to contract and collapse depending on the actual $\mathrm{pH}$ of the medium. ${ }^{14,15}$ In the electrolyte diodes developed for analytical purposes, hydrogels are commonly utilized as connecting elements between the acidic and alkaline reservoirs. ${ }^{16,17}$ Environmental-sensitive hydrogels are frequently used materials not only in chemistry and physics but also in biomedical applications, such as tissue 
engineering and drug delivery. ${ }^{18,19}$ For those kinds of applications, the improvement of shear-thinning and self-healing properties together with a reduced response time is essential and may be tuned by changes in $\mathrm{pH}$ or temperature..$^{20-22}$ The case of $\mathrm{pH}$ change links again the diffusion properties of hydrogen ions in the actual gel.

For each of those purposes, the quality and concentration of the gel alongside with additional substances can remarkably affect the diffusion of chemical species. Regarding pattern formation, the modified diffusion can be tracked via different pattern morphologies in other types of reaction-diffusion systems such as Liesegang patterns. ${ }^{23,24}$ Empirical evidence implies that the diffusion rate of chemical species - in particular that of $\mathrm{H}^{+}$- may reasonably change in hydrogels compared to dilute aqueous solutions due to structural or chemical interactions; however, the extent of this change cannot be trivially inferred. So far in numerical studies attempting to model pattern formation in acid auto-activated systems, only theoretical values and approximations have been available for the diffusion coefficients of the relevant species in hydrogels. Therefore our aim is to provide practical values for ionic diffusion coefficients, particularly $D_{\mathrm{H}^{+}}$ under frequent experimental conditions.

Special attention has to be given to finding the most appropriate experimental technique due to the challenging circumstances. Several well-established and accessible techniques may be mentioned for determining diffusion coefficients e.g. using radioactive tracers, ${ }^{25}$ fluorescence methods, ${ }^{26,27}$ holographic laser interferometry, ${ }^{28}$ the refractive index method, ${ }^{29}$ nuclear magnetic resonance techniques, ${ }^{30}$ etc. Evidently, radioactive and fluorescence methods are not suitable for following the movement of hydrogen ions; furthermore, in occasionally opalescent hydrogels, such as agarose or agar-agar, the efficiency of interferometric and refractive methods may be significantly reduced in the case of small ions, even though these techniques have been reliably used for studying larger species such as protein macromolecules. Pulsed-field gradient spin echo (PFGSE) NMR spectroscopy can be readily applied to measure a large number of small individual ions including ${ }^{1} \mathrm{H}$ nucleus; ${ }^{31,32}$ however, hydrogel media generate excessive water signals that cannot be suppressed without eliminating the relevant signal of free protons as well.

The scientific problem, namely to determine relevant individual diffusion coefficients of ions experimentally if the surroundings also contain the same ion in the immobilized form, is not solved yet. Therefore, as we present the particular case of the hydrogen ion in agarose gel, we have developed a complex experimental procedure as a combination of available and effective techniques. This paper introduces our methods and demonstrates the experimental results accompanied by numerical modeling of ionic diffusion.

For an easier understanding we present the rather complex results in separate parts regarding either the relevant experimental techniques or the performed modeling. First we introduce the theoretical background of the method including the basis of conductance measurements and of PFGSE-NMR. We then describe the actual conductometric cell followed by its electric properties and the diffusion-migration model.
We come to the experimental section which contains two major parts:

(1) Conductance measurements to obtain relevant effective diffusion coefficients for electrolytes;

(2) PFGSE-NMR measurements to obtain individual diffusion coefficients.

In the end, we combine all those data in Section 6 to present our final results considering the diffusion coefficient of the hydrogen ion in agarose gel.

\section{Theoretical background}

Among many other techniques, a conductance method was developed by Harned and French for the purpose of determining the effective diffusion coefficient ( $D_{\text {eff }}$ ) of electrolytes in dilute aqueous solutions. ${ }^{33-38}$ They aimed for maximum simplicity concerning the mathematical treatment of the experimental data. Here we recall some of their results and formulation for easier understanding.

The diffusional cell in the shape of a cuvette with length $a$ contained two pairs of platinum electrodes placed at a distance $\xi$ from both ends (see Fig. 1). The medium confined by the cuvette can be considered one dimensional, for which Fick's second law

$$
\frac{\partial c}{\partial t}=D \frac{\partial^{2} c}{\partial x^{2}}
$$

with no flux boundary conditions $\frac{\partial c}{\partial x}=0$ at $x=0$ and $x=a$ has a solution in the form of a Fourier cosine series as

$$
c=\sum_{n=0}^{\infty} A_{n} \exp \left(-\frac{n^{2} \pi^{2} D}{a^{2}} t\right) \cos \frac{n \pi x}{a},
$$

where $c$ is the concentration of the diffusing species and $D$ is its diffusion coefficient, $A$ is a Fourier coefficient, $a$ is the physical height of the cuvette, $x$ is the coordinate in the direction of

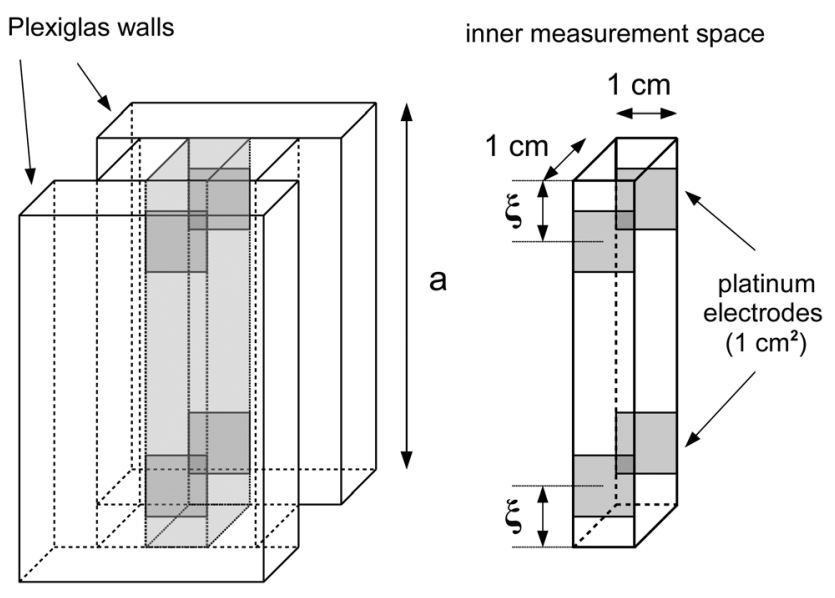

Fig. 1 Design of the conductometric cell: the height of the inner space is $a=6 \mathrm{~cm}$, the center of each square shaped platinum electrode embedded in the Plexiglas walls is placed $\xi=1 \mathrm{~cm}$ far from the top or bottom of the cell. The inner space is filled with a $6 \mathrm{~cm}^{3}$ total volume of the hydrogel. 
diffusion, and $t$ is time. The expression for the difference in electrolyte concentration between the top $(\xi)$ and bottom $(a-\xi)$ electrodes is then written as

$$
\begin{aligned}
c(\xi)-c(a-\xi)= & 2 A_{1} \exp \left(-\frac{\pi^{2} D t}{a^{2}}\right) \cos \frac{\pi \xi}{a} \\
& +2 A_{3} \exp \left(-9 \frac{\pi^{2} D t}{a^{2}}\right) \cos \frac{3 \pi \xi}{a}+\ldots,
\end{aligned}
$$

which only contains odd terms. Moreover, Harned and French chose $\xi=\frac{a}{6}$ cell geometry eliminating the term with $n=3$. The right-hand side is then reduced to a more simplified form, given that after sufficient time - due to the rapid convergence of the higher order exponentials - only the first term will have significant contribution, leading to

$$
c\left(\frac{a}{6}\right)-c\left(\frac{5 a}{6}\right)=A \exp \left(-\frac{\pi^{2} D t}{a^{2}}\right),
$$

where $A=2 A_{1} \cos \frac{\pi}{6}$.

In dilute solutions, the difference $c\left(\frac{a}{6}\right)-c\left(\frac{5 a}{6}\right)$ can be considered to be proportional to the difference in specific conductivity $\kappa$ at the same positions, i.e., $C_{\text {top }} G_{\text {top }}-C_{\text {bottom }} G_{\text {bottom }}$, where $C_{\text {top }}$ and $C_{\text {bottom }}$ are the cell constants regarding the upper and the lower pairs of electrodes, while $G$ is the measured conductance. When this difference in $G$ is substituted into eqn (4), a direct formula

$$
G_{\text {top }}-\frac{C_{\text {bottom }}}{C_{\text {top }}} G_{\text {bottom }}=\mathscr{C} \exp \left(-\frac{\pi^{2} D}{a^{2}} t\right),
$$

where $\mathscr{C}$ stands for the preexponential coefficient, is obtained that allows the determination of diffusion coefficients directly from conductance values. Linearization

$$
\ln \left(G_{\text {top }}-\frac{C_{\text {bottom }}}{C_{\text {top }}} G_{\text {bottom }}\right)=-\frac{\pi^{2} D}{a^{2}} t+\ln \mathscr{C}
$$

is hence readily applicable for the easier visualisation of the experimental results. The advantage of this treatment lies in the invariance to the initial concentration distribution as long as a difference in the concentration at the two positions $(\xi$ and $(a-\xi))$ is established at $t=0$ without allowing large concentrations. Therefore, eqn (5) is one of the two particularly important equations for the evaluation of the conductance measurements.

The other essential equation relies in the fact that the diffusion coefficients of individual ions cannot be determined directly using this method, since all ionic components in the system contribute to conductance. However, a simple formula exists for the effective diffusion coefficient $\left(D_{\text {eff }}\right)$ for $1: 1$ binary strong electrolytes

$$
D_{\text {eff }}=\frac{2 D_{\mathrm{A}} D_{\mathrm{C}}}{D_{\mathrm{A}}+D_{\mathrm{C}}}
$$

where $D_{\mathrm{A}}$ and $D_{\mathrm{C}}$ stand for the individual diffusion coefficients of the anion and the cation, respectively. Provided that $D_{\text {eff }}$ of an electrolyte containing $\mathrm{H}^{+}$as the cation is determined, the individual diffusion coefficient of hydrogen ions can only be calculated using eqn (7) if $D_{\mathrm{A}}$ is obtained from independent measurements.

One experimental technique capable of measuring individual ionic diffusion coefficients is based on PFGSE-NMR spectroscopy. ${ }^{31,32}$ In the presence of a magnetic field gradient $g$, an exponentially decaying signal $I(g)$ can be detected according to the Stejskal-Tanner equation:

$$
I(g)=I(0) \exp \left(-\gamma^{2} g^{2} \delta^{2} D\left(\Delta-\frac{\delta}{3}-\frac{\tau}{2}\right)\right)
$$

where $I_{0}$ is the signal in the absence of the field gradient, $\gamma$ is the gyromagnetic ratio of the measured nucleus, $\delta$ is the gradient pulse length, $\Delta$ is the delay time between gradient pulses, $\tau$ is the delay time of the radio frequency pulses, and finally $D$ is the individual diffusion coefficient of the corresponding species containing the detected nuclues. No concentration gradient and hence no diffusional potential develop during the NMR measurements; therefore ionic species diffuse independently of each other. For later on, the evaluation of our NMR measurements is based on eqn (8).

Although PFGSE-NMR spectroscopy cannot be directly used for measuring the diffusion coefficient of $\mathrm{H}^{+}$due to the excessive water signal in hydrogels, it is available for other small ions with NMR-active nuclei. Therefore, the isotope ${ }^{23} \mathrm{Na}$ is chosen to obtain an individual $D_{\mathrm{C}}$ value in eqn (7) for sodium ions, while conductance measurements are performed for $\mathrm{NaCl}$ and $\mathrm{HCl}$ solutions - electrolytes with a common anion - under the same conditions. To combine all results, eqn (7) is applied twice, first to calculate $D_{\mathrm{Cl}^{-}}$from $D_{\mathrm{Na}^{+}}$(via NMR) and $D_{\text {eff, } \mathrm{NaCl}}$ (via conductance), then a second time using $D_{\mathrm{Cl}^{-}}$(from the previous step) and $D_{\text {eff,HCl }}$ (via conductance) to determine the final diffusion coefficient of hydrogen ions.

To verify the selection of the ${ }^{23} \mathrm{Na}$ nucleus, the authors note that hydrogen chloride solution may be chosen for conductance measurements as a suitable electrolyte that contains $\mathrm{H}^{+}$ as the cation; however, the properties of NMR active chlorine isotopes such as their relaxation times make chloride ions unfavorable for PFGSE-NMR measurements.

\section{Cell design}

For determining the required effective diffusion coefficients of sodium chloride and hydrogen chloride in a hydrogel, we have designed a conductometric cell based on the original concept of Harned and French. ${ }^{33}$ The height of the cell $a=6 \mathrm{~cm}$ while its cross section is $1 \mathrm{~cm} \times 1 \mathrm{~cm}$. This inner measurement space enclosed by Plexiglas walls was constructed with an open top and bottom face that could be filled with the hydrogel and then sealed airtight, as illustrated in Fig. 1.

To measure the change of conductance while an adjusted concentration gradient of the main diffusing electrolyte diminishes with time, four pieces of square shaped $1 \mathrm{~cm}^{2}$ platinum foils were embedded in the Plexiglas walls opposite to each other thus forming two pairs of electrodes. The center of each electrode was located $\xi=1 \mathrm{~cm}$ from both the top and 
bottom ends. These $a$ and $\xi$ values hence correspond to the $\xi=\frac{a}{6}$ ratio requirement introduced by Harned and French and therefore allow us to use eqn (5) for the evaluation in our experiments. For consistent measurements, the cell was always positioned in the same orientation and the ends were marked permanently in order for the electrode pairs to become distinguishable as the top pair and the bottom pair (as referred to henceforth). Each embedded platinum electrode was soldered on a copper rod to which the electric wires could be attached during the measurements.

\section{Modeling}

During conductance measurements, two major risks may arise intrinsically originating in the method. First, the measuring electrodes are extended surfaces $\left(1 \mathrm{~cm}^{2}\right)$ and not point objects. Therefore, during the calculations, we have validated the substitution of molar conductivities at the preset locations $x=a / 6$ and $x=5 a / 6$, related to the concentrations in eqn (4), with those obtained from the measurements in the electrode areas facing an inhomogeneous concentration distribution with regards to the main diffusing electrolyte.

Second, in hydrogels a considerable amount of ions can be present inherently in homogeneous concentration distribution depending on the chemical properties of the polymer. Those ions may be regarded as a background electrolyte where ionic components may be free hydrated ions or charged functional groups of the polymer chain. Since all ionic components contribute to the conductance measured and thus to the effective diffusion coefficient obtained, the presence of a background electrolyte may render eqn (7) invalid for the purpose of calculating $D_{\mathrm{H}^{+}}$. We have therefore decided to thoroughly investigate the effect of a background electrolyte on the resulting $D_{\text {eff }}$ when different parameters, such as the diffusion coefficients of ions or the concentration of the background electrolyte, are varied.

Let us now present how the model is built and validated with regards to the technical issues mentioned before. To do so, we consider a model system having dimensions equivalent to the experimental cell. An electrolyte with several sets of properties (concentration, diffusion coefficient, etc.) diffuses through the medium due the concentration gradient maintained between the two ends of the cell as the initial condition. The diffusive spreading is tracked via the change of conductivity calculated between electrode surfaces.

\subsection{Diffusion of ions}

The conductometric cell can be considered one dimensional along its long axis. In this case the transport of ions can be described by the component and the charge balance equations according to

$$
\begin{gathered}
\frac{\partial C_{i}}{\partial t}=D_{i} \frac{\partial^{2} C_{i}}{\partial x^{2}}+\frac{z_{i} F D_{i}}{R T} \frac{\partial}{\partial x}\left(C_{i} \frac{\partial \Psi}{\partial x}\right) \\
\frac{\partial Q}{\partial t}=\sum_{i=1}^{n}\left(z_{i} F D_{i} \frac{\partial^{2} C_{i}}{\partial x^{2}}+\frac{z_{i}^{2} F^{2} D_{i}}{R T} \frac{\partial}{\partial x}\left(C_{i} \frac{\partial \Psi}{\partial x}\right)\right)=0
\end{gathered}
$$

where $C_{i}, z_{i}$ and $D_{i}$ are the concentration, the charge, and the diffusion coefficient of the $i$ th component, respectively, while $\Psi$ is the electric potential. ${ }^{39,40}$ In eqn (9) the first term on the right-hand side expresses diffusional flux, while the second one represents ionic migration in an inhomogeneous electric field. The charge balance in eqn (10) states that no macroscopic charge separation may occur in the system under our conditions. For simpler computational treatment, eqn (9) and (10) are transformed into dimensionless forms

$$
\begin{gathered}
\frac{\partial c_{i}}{\partial \tau}=\delta_{i} \frac{\partial^{2} c_{i}}{\partial \xi^{2}}+z_{i} \delta_{i} \frac{\partial}{\partial \xi}\left(c_{i} \frac{\partial \psi}{\partial \xi}\right) \\
0=\sum_{i=1}^{n}\left(z_{i} \delta_{i} \frac{\partial^{2} c_{i}}{\partial \xi^{2}}+z_{i}^{2} \delta_{i} \frac{\partial}{\partial \xi}\left(c_{i} \frac{\partial \psi}{\partial \xi}\right)\right)
\end{gathered}
$$

applying the dimensionless parameters $\tau=\frac{D}{L_{0}^{2}} t, \quad c_{i}=\frac{C_{i}}{C_{0}}$, $\xi=\frac{x}{L_{0}}$, and $\psi=\frac{F}{R T} \Psi$, where $D$ is the diffusion coefficient of the diffusing anion, $C_{0}$ is the input concentration of the diffusing electrolyte at one end, and $L_{0}=5 \times 10^{-3} \mathrm{~cm}$. Onedimensional spatial discretisation is then applied using the central difference formula for the first derivatives and the standard three-point Laplacian for the second derivatives. Introducing no-flux boundary conditions, the initial value problem defined by the discretised component balances is solved using backward differential formulae. The potential is calculated in each iteration step by solving the linear algebraic equation defined by the discretised charge balance.

The iterations are carried out on 12001 grid points with spatial resolution $\Delta x=0.1$ using the CVODE package. The conductivity at each dimensionless coordinate is evaluated according to $\kappa_{j}=\sum_{i=1}^{n} z_{i}^{2} \delta_{i} c_{i, j}$, then an average value is calculated around points $\xi=200$ and $\xi=1000$ corresponding to the locations of the electrodes. These average values ( $\kappa_{\text {top }}$ and $\left.\kappa_{\text {bottom }}\right)$ are then used to obtain the effective diffusion coefficient by fitting

$$
\kappa_{\text {top }}-\kappa_{\text {bottom }}=\mathscr{K} \exp \left(-\frac{\pi^{2} \delta_{\text {eff }}}{\alpha^{2}} \tau\right)
$$

i.e., the dimensionless form of eqn (5) provided that conductivity $\kappa$ is proportional to conductance $G$.

Two reference cases with different ratios of the diffusion coefficients of the cation and the anion have been selected for a binary electrolyte of ion charges $z_{1}=1$ and $z_{2}=-1$. The first case with $\delta_{1}=7, \delta_{2}=1$ corresponds to the scenario in water where hydrogen ions can diffuse approximately seven times faster than other small ions due to the Grotthuss mechanism. The second case with $\delta_{1}=3, \delta_{2}=1$ anticipates the deceleration of the hydrogen ions in hydrogels compared to dilute solutions.

Calculations have been performed for a second set of conditions where a background electrolyte is introduced with a cation $\left(z_{3}=1, \delta_{3}=1\right)$ and an anion $\left(z_{4}=-1\right.$ or $-2, \delta_{4}=1$ or 0$)$. The last case with $\delta_{4}=0$ represents a hydrogel that contains bound anionic functional groups (e.g., carboxylate groups in gelatin). 
As initial conditions, the concentration profile of the ions of the main electrolyte is set according to $c_{1}=c_{2}=1$ for $\xi \leq 10$ and $c_{1}=c_{2}=0$ for $10<\xi \leq 1200$. Meanwhile, the relative concentration of the homogeneously distributed background electrolyte is varied as $10^{-1}, 10^{-2}, 10^{-3}, 10^{-4}$, and $10^{-5}$ with respect to the input concentration of the main electrolyte at the boundary.

The temporal evolution of $\kappa_{\text {top }}$ and $\kappa_{\text {bottom }}$ is presented in Fig. 2(a) for a selected case with no background electrolyte. The black dashed curve corresponding to the upper electrode near the area with high electrolyte concentration reveals an increase of conductivity caused by the incoming ions after the beginning of diffusion. After a short transient period, where $\kappa_{\text {top }}$ has a local maximum, the conductivity decreases due to the depletion of the zone. Meanwhile, $\kappa_{\text {bottom }}$ increases thanks to the incoming ions of the diffusing electrolyte. Plotting $\left(\kappa_{\text {top }}-\kappa_{\text {bottom }}\right)$ versus the non-dimensional time depicts an exponential decay following a short transient period which is shown in Fig. 2(b) in a logarithmic form for easier visualisation. The $\delta_{\text {eff }}$ value of the main electrolyte can be obtained with the standard error of less than $0.01 \%$ using eqn (13). For the case of $\delta_{1}=3$ and $\delta_{2}=1$ we obtain 1.500, while for $\delta_{1}=7$ and $\delta_{2}=1$ we have 1.750 (without background electrolyte in both cases), indicating that these values are in excellent agreement with the theoretical ones
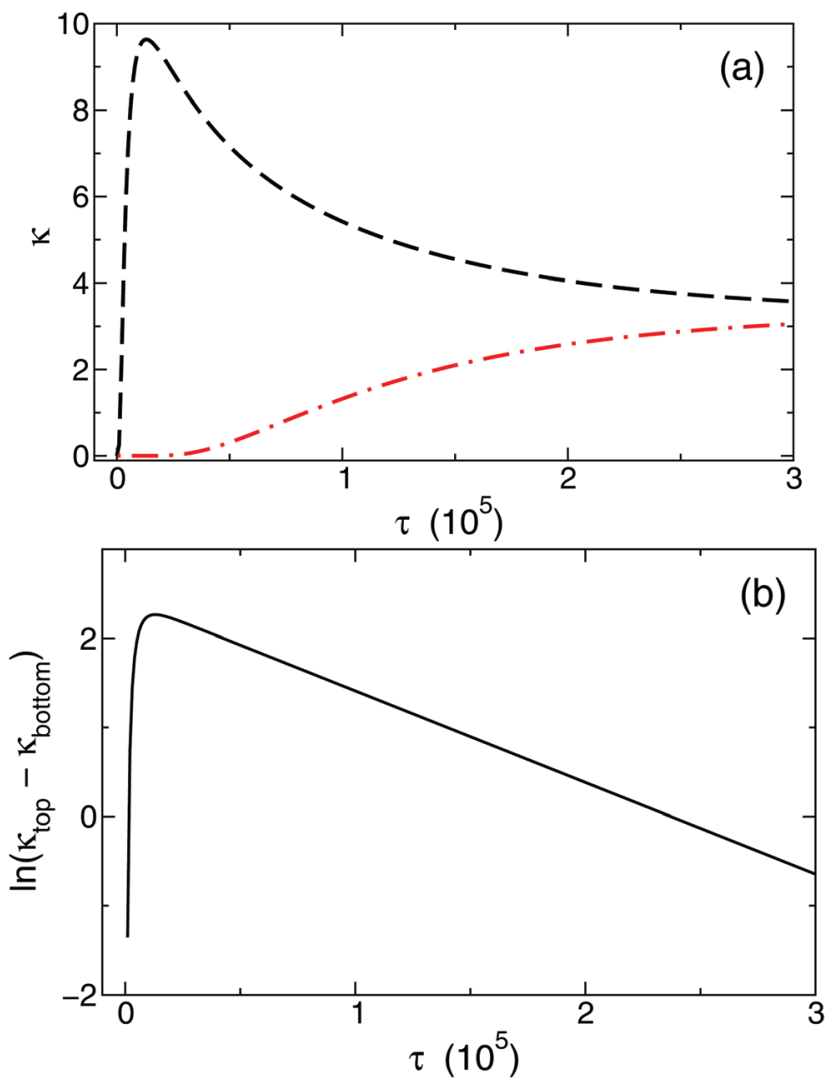

Fig. 2 Temporal evolution of the calculated mean conductivity in the absence of background electrolyte at the top (black dashed curve) and bottom (red dashed and dotted curve) electrodes (a). Logarithm of the conductivity difference resulting in a linear correlation (b). obtained from eqn (7): $\frac{3}{2}$ and $\frac{7}{4}$, respectively. Therefore, with the local input of the electrolyte in one end of the conductometric cell, the higher order Fourier terms indeed decay fast making eqn (4) valid. In addition, this also means that the conductivities at the ideal point locations preset according to the model can be replaced with the spatially averaged values relevant to experimental measurements.

Even though an exponential decay is still observed in the model introducing a background electrolyte with increasing concentration compared to the main electrolyte, the determined values for $\delta_{\text {eff }}$ exhibit an increasing deviation from those valid for pure binary electrolytes according to eqn (7). The influence of a background electrolyte can also be observed graphically as shown in Fig. 3. The resulting logarithmic curves for two different calculations are displayed where every parameter was kept constant except for the relative concentration of the background electrolyte. Therefore, a relative concentration exists, above which the exponential correlation required for eqn (13) becomes perceptibly distorted. The deviation from the ideal behavior can be tracked by changing the background electrolyte concentration provided that all other parameters are kept constant. Several values of $\delta_{\text {eff }}$ are listed in Table 1 . After a systematic mapping of the parameters, we have found that the application of eqn (7) for calculating individual diffusion coefficients is acceptable for background electrolyte concentrations not more than $10^{-4}$ of the main diffusing binary electrolyte. This leads to an important experimental restriction, namely that in any experiment this large concentration difference has to be maintained to collect data applicable for evaluation.

\subsection{Electric properties of the conductometric cell}

In Harned and French's method, the conductance and hence conductivity values expressed at an $\frac{a}{6}$ distance from top and bottom of the cell are used for evaluation. In our custom-made

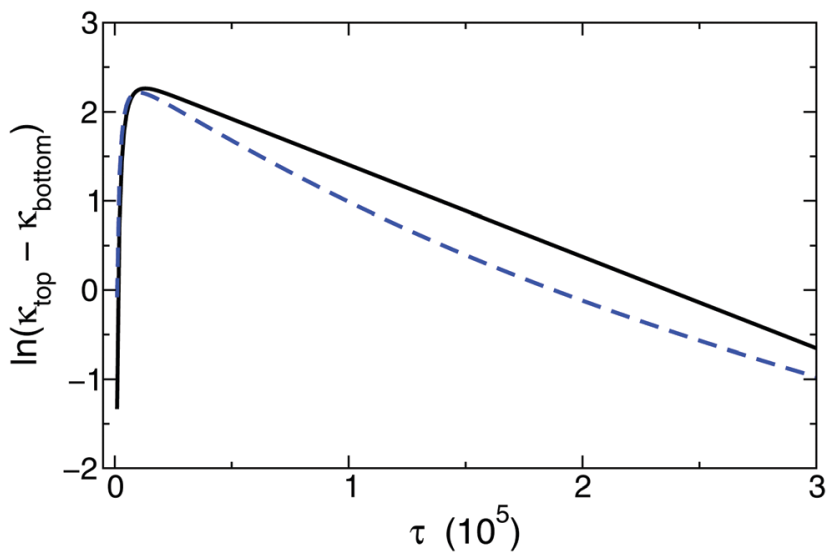

Fig. 3 Graphical illustration of the impact of background electrolyte concentration on ionic diffusion. The black solid line represents a case when the relative concentration of the background electrolyte is low enough $\left(10^{-4}\right)$ to be negligible, meanwhile the blue dashed curve was acquired with a high concentration ratio $\left(10^{-1}\right)$ resulting in distorted correlation. 
Table 1 Effective diffusion coefficients determined for a selected case $\left(\delta_{1}=3, \delta_{2}=\delta_{3}=1, \delta_{4}=0, z_{1}=z_{2}=z_{3}=1, z_{4}=-2\right)$ with increasing background electrolyte concentration $\left(c_{b}\right)$. The standard errors for $\delta_{\text {eff }}$ values are less than $0.1 \%$

\begin{tabular}{ll}
\hline$c_{\mathrm{b}}$ & $\delta_{\text {eff }}$ \\
\hline $10^{-5}$ & 1.499 \\
$10^{-4}$ & 1.491 \\
$10^{-3}$ & 1.356 \\
$10^{-2}$ & 1.092 \\
$10^{-1}$ & 1.019 \\
\hline
\end{tabular}

cell, the electrodes are centered at these locations; however, their $1 \mathrm{~cm}^{2}$ area is not negligible considering the concentration gradient developing during the experiments.

Solution of the differential form of Ohm's law for our custom-made cell reveals that the electric current patterns between the two electrode surfaces in a pair are close to ideal parallel forms and, therefore, average conductance measured this way may be validly used for obtaining effective diffusion coefficients.(for details see the ESI, $†$ Section A)

\section{Experimental}

\subsection{Sample preparation for impedance and conductance measurements}

Our experiments designed to determine the diffusion coefficient of hydrogen ions were conducted in agarose hydrogel from which $2 \mathrm{w} / \mathrm{v} \%$ samples were prepared in order to fill the $6 \mathrm{~cm}^{3}$ inner space of the conductometric cell. This setup (see Fig. 1) was used both for impedance (see ESI, $\dagger$ Section B) and conductance measurements.

For gel preparation, analytical reagent agarose powder (VWR DNA Grade) was mixed with deionized water to create a $2 \mathrm{w} / \mathrm{v} \%$ solution, then the mixture was stirred and heated in a covered beaker until boiling. The homogeneous solution was left to cool for a few minutes, with constant stirring, then filled into the conductometric cell. Finally, it was left to set at room temperature. Later, the inner space of the cell was closed airtight, the cell was assembled and placed in a thermostated container for at least 12 hours prior the initiation. Meanwhile, a separate $2 \mathrm{~mm}$ thick, $1 \mathrm{~cm} \times 1 \mathrm{~cm}$ piece of agarose was also prepared using a Plexiglas frame. The separate piece of gel was immersed in either $1 \mathrm{M}$ $\mathrm{NaCl}$ or $\mathrm{HCl}$ solution (Molar) for the same time and used later to initiate diffusion.

\subsection{Conductance measurements}

Conductance measurements have been carried out inside a thermostated container at $25{ }^{\circ} \mathrm{C}$ while temperature fluctuation is constantly monitored throughout the experiment. The cell is electrically connected to the conductometer through a relay allowing a switch between the top and bottom electrodes in order to avoid cross-currents arising while simultaneously measuring at both pairs. Each conductance measurement is initiated by reopening the cell and placing the small piece of gel impregnated by electrolyte solution on top of the $6 \mathrm{~cm}^{3}$ agarose already filled and set in the inner space of the conductometric cell.
The cell is then closed again and placed in the thermostated container for 90 minutes to let the main diffusing electrolyte penetrate. When the allotted time is over, the small hydrogel piece is removed, the cell is resealed and the measurement is initiated. Conductance is recorded first at the top then, after a switch in the relay, at the bottom electrodes. After two-minute pause, the conductance is measured again in the same manner. This cycle is repeated over the next 3-5 days and the data are collected using a computer. (For the validation of conductance measurements in the custom-made cell see the ESI, $†$ Section B.)

\subsection{PFGSE-NMR measurements}

The gel preparation process for PFGSE-NMR samples is similar to that for conductance measurements. A $0.1 \mathrm{M} \mathrm{NaCl}$ solution is made prior to gel preparation with $10 \mathrm{v} / \mathrm{v} \% \mathrm{D}_{2} \mathrm{O}$ (heavy water) content instead of pure deionised water, and then used for making a $2 \mathrm{w} / \mathrm{v} \%$ agarose mixture. After stirring and heating the solution, a preheated quartz NMR tube is filled with a small amount of the solution. Then the tube is closed and the gel is allowed to cool and set.

PFGSE-NMR measurements are performed on a Bruker Avance DRX $500 \mathrm{MHz}$ instrument tuned to ${ }^{23} \mathrm{Na}$ nucleus at $25{ }^{\circ} \mathrm{C}$. The spectral width is set to $4000 \mathrm{~Hz}$ while the added $\mathrm{D}_{2} \mathrm{O}$ is used for locking the signal. The LED (longitudinal eddy-current delay) pulse sequence is applied where $\delta$ pulse length for the gradient field is set to $22000 \mu \mathrm{s}$ with $\Delta=0.03 \mathrm{~s}$ and $\tau=0 \mathrm{~s}$ delay times between pulses (see eqn (8)).

\section{Results and discussion}

In the previous sections, it has been shown that an exponential relationship based on eqn (13) may be used to determine effective diffusion coefficients of binary electrolytes via conductance measurements provided that the concentration of the background electrolyte present in the hydrogel is sufficiently small. In addition, it has also been shown that eqn (7) may be validly used for such conditions to derive individual diffusion coefficients. We have determined both the theoretical and experimental cell constants in satisfactory agreement. The custom-made conductometric cell working with alternating current has proved to deliver impedance data where ohmic resistance is the major component (see ESI $\dagger$ ). These requirements listed enable us to link together all experimental results and come to the final conclusions.

For each recorded conductance value, conductivity is calculated using the experimental cell constants $C_{\text {top }}$ and $C_{\text {bottom. }}$. Conductance measurements are evaluated using the dimensional form of eqn (13) as

$$
\kappa_{\text {top }}-\kappa_{\text {bottom }}=\mathscr{C} \exp \left(-\frac{\pi^{2} D_{\text {eff }}}{a^{2}} t\right)
$$

for which logarithmic transformation is applied for clearer visualisation of the validity of exponential fitting. Our main results are depicted in Fig. 4, which shows the recorded and transformed experimental data for one example of the performed conductance measurements. The typical temperature 

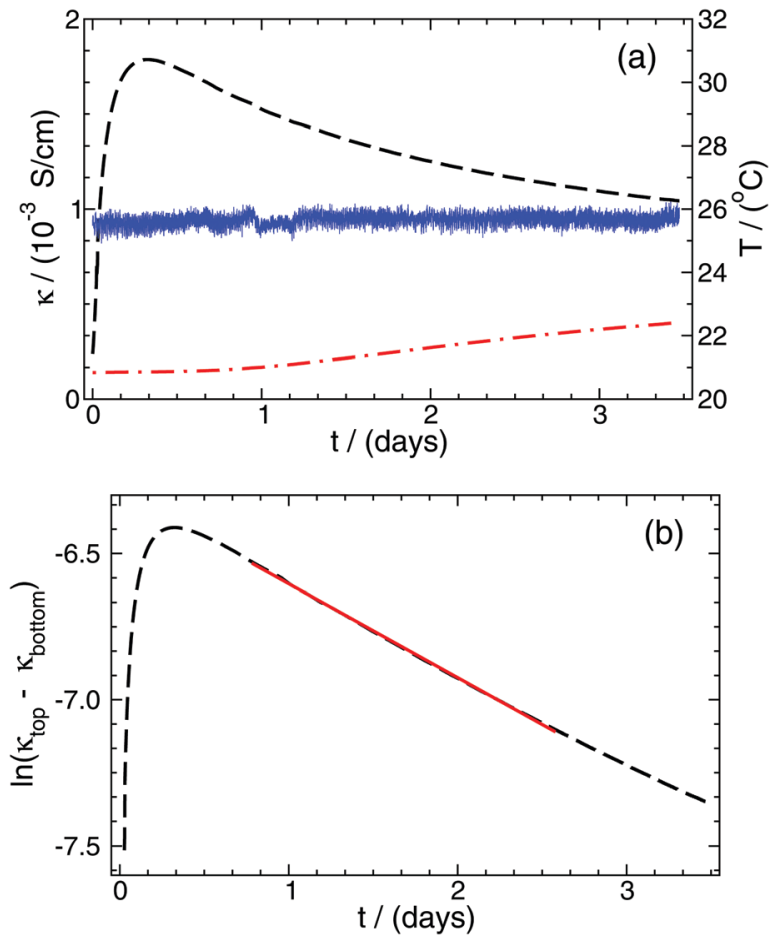

Fig. 4 Conductivity $(\kappa)$, calculated from the measured conductance and the appropriate cell constant, as a function of time at the top (black dashed curve) and bottom (red dashed and dotted curve) electrode pairs, respectively (a). The thickness of the line connecting the data points is greater than the fluctuations in the conductivity due to the $0.5 \%$ precision of the measurements. The solid blue curve represents the temperature recorded throughout the experiment. The logarithm of the conductivity difference between the two electrode pairs as a function of time (b). The solid red line represents the function fitted to the most appropriate time interval according to eqn (14).

fluctuation is also shown in blue in panel (a) which is considered to be sufficiently small not to influence significantly the measured conductance values. The trends of the curves are similar to those seen during the modeling in Fig. 2; the conductivity at the upper electrode steeply increases at the beginning when the main diffusing electrolyte penetrates the region. Meanwhile, no change is seen at the lower electrode. At later stages the concentration gradient of the electrolyte decays via diffusion, we therefore observe a slow decrease of $\kappa_{\text {top }}$ with a

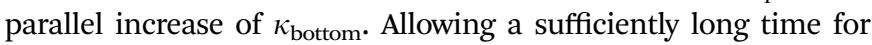
the experiment to reach homogeneous concentration distribution, no further change of $\kappa$ would be detected.

To determine the effective diffusion coefficient of the actual electrolyte, the function in eqn (14) is fitted to the data set obtained over a two-day period; its linearised form is presented in Fig. 4(b) for easier visualisation. This example case shows that an exponential decay is recovered in the experiments in good agreement with the theory detailed earlier. The effective diffusion coefficients $D_{\text {eff }}$ for $\mathrm{NaCl}$ and $\mathrm{HCl}$ electrolytes are calculated as an average of two or three parallel measurements given that $a=6 \mathrm{~cm}$. Then, from PFGSE-NMR measurements, an individual value for the diffusion coefficient of $\mathrm{Na}^{+}$ions is obtained using the Stejskal-Tanner equation (eqn (8)) for exponential curve-fitting. By applying eqn (7) twice - first for
Table 2 Effective $\left(D_{\text {eff }}\right)$ and individual $(D)$ diffusion coefficients determined experimentally in $2 \mathrm{w} / \mathrm{v} \%$ agarose gel by conductance and PFGSENMR measurements

\begin{tabular}{lc}
\hline Diffusion coefficient & Value $\left(10^{-5} \mathrm{~cm}^{2} \mathrm{~s}^{-1}\right)$ \\
\hline$D_{\text {eff, } \mathrm{NaCl}}$ & $1.640 \pm 0.001$ \\
$D_{\text {eff,HCl }}$ & $3.141 \pm 0.002$ \\
$D_{\mathrm{Na}^{+}}$ & $1.35 \pm 0.02$ \\
$D_{\mathrm{Cl}^{-}}$ & $2.10 \pm 0.04$ \\
$D_{\mathrm{H}^{+}}$ & $6.22 \pm 0.37$ \\
\hline
\end{tabular}

$D_{\mathrm{Na}^{+}}$and $D_{\text {eff,Nacl }}$ to obtain $D_{\mathrm{Cl}^{-}}$, then a second time for $D_{\mathrm{Cl}^{-}}$ and $D_{\text {eff,HCl }}$ to obtain $D_{\mathrm{H}^{+}}$- the final individual diffusion coefficients of $\mathrm{Cl}^{-}$and $\mathrm{H}^{+}$ions are determined in $2 \mathrm{w} / \mathrm{v} \%$ agarose hydrogel. All those results are summarized in Table 2.

The effect of a hydrogel medium on the diffusion of electrolytes and single ions can be concluded from our results as follows. In agarose, the mobility of simple ions such as $\mathrm{Cl}^{-}$and $\mathrm{Na}^{+}$does not decrease significantly compared to their diffusion coefficients measured in dilute aqueous solutions $\left(D_{\text {eff }, \mathrm{NaCl}}=\right.$ $1.586 \times 10^{-5} \mathrm{~cm}^{2} \mathrm{~s}^{-1}$ taken from the results of Harned and Hildreth ${ }^{37}$ ), which implies that they can freely diffuse in the high water content of the hydrogel. This also shows that there is no significant charge on the polymeric chain of the gel. However, the eventual $D_{\mathrm{H}^{+}}$value is only 3-4 times greater than the coefficients of other small ions, which supports the idea that, due to structural interactions between the polymer chain and the diffusing ions, the movement of hydrogen ions is hindered. It is probable that the spatial arrangement of polymer chains interferes with the hopping protons and thus reduces the efficiency of the Grotthuss mechanism. In hydrogels containing counterions, the polyelectrolyte backbone can further hinder the diffusion of hydrated hydrogen ions. ${ }^{41}$ It is our intention to further investigate the diffusion of hydrogen ions (and possibly the hydroxide ion as well) in such hydrogels that are widely used in the studies of chemical pattern formation and other applications listed in Section 1.

\section{Conclusion}

The diffusion coefficient of hydrogen ion in hydrogels plays an important role in many research activities, e.g. in reaction-diffusion pattern formation, $\mathrm{pH}$ responsive gels, $\mathrm{pH}$-regulated selfhealing mechanisms, and in hydrogels used for drug delivery. Their values, however, have only been approximated lacking a method that can directly deliver the relevant data. Here we have presented a complex but reproducible experimental procedure based on conductance and PFGSE-NMR measurements to gain access to the desired diffusion coefficient. With modeling calculations we have also shown that this general method can be extended to determine the relevant diffusion coefficients of the hydrogen ion in different hydrogel media.

\section{Acknowledgements}

This work was supported by the National Research, Development and Innovation Office (K119795) and GINOP-2.3.2-15-2016-00013 
projects. G. Schuszter also thanks the National Research, Development and Innovation Office (PD121010), while T. Gehér-Herczegh thanks ESTEC/4000102255/11/NL/KLM for the financial support.

\section{References}

1 Q. Ouyang and H. L. Swinney, Nature, 1991, 352, 610-612.

2 J. Horváth, I. Szalai and P. De Kepper, Science, 2009, 324, 772-775.

3 I. Szalai and P. De Kepper, Comm. Pure \& Appl. Anal., 2012, 11, 189-207.

4 J. E. Pearson, Science, 1993, 261, 189-192.

5 K.-J. Lee, W. D. McCormick, J. E. Pearson and H. L. Swinney, Nature, 1994, 369, 215-218.

6 Z. Virányi, I. Szalai, J. Boissonade and P. De Kepper, J. Phys. Chem. A, 2007, 111, 8090-8094.

7 I. Molnár and I. Szalai, J. Phys. Chem. A, 2015, 119, 9954-9961.

8 I. Szalai, J. Horváth, N. Takács and P. De Kepper, Phys. Chem. Chem. Phys., 2011, 13, 20228-20234.

9 A. Hanna, A. Saul and K. Showalter, J. Am. Chem. Soc., 1982, 104, 3838-3844.

10 D. Horváth and A. Tóth, J. Chem. Phys., 1998, 108, 1447-1451.

11 M. Fuentes, M. N. Kuperman and P. De Kepper, J. Phys. Chem. A, 2001, 105, 6769-6774.

12 M. Dayeh, M. Ammar and A. G. Mazen, RSC Adv., 2014, 4, 60034-60038.

13 I. Szalai, J. Phys. Chem. A, 2014, 118, 10699-10705.

14 I. Varga, I. Szalai, R. Mészaros and T. Galányi, J. Phys. Chem. B, 2006, 110, 20297-20301.

15 T. Ueki and R. Yoshida, Phys. Chem. Chem. Phys., 2014, 16, 10388-10397.

16 L. Roszol, T. Lawson, V. Koncz, Z. Noszticzius, M. Wittmann, T. Sarkadi and P. Koppa, J. Phys. Chem. B, 2010, 114, 13718-13725.

17 L. Roszol, A. Várnai, B. Lorántfy, Z. Noszticzius and M. Wittmann, J. Chem. Phys., 2010, 132, 064902.

18 Y. Qiu and K. Park, Adv. Drug Delivery Rev., 2001, 53, 321-339.

19 T. Manouras and M. Vamvakaki, Polym. Chem., 2017, 8, 74-96.
20 V. Yesilyurt, J. M. Webber, E. A. Appel, C. Godwin, R. Langer and D. G. Anderson, Adv. Mater., 2016, 28, 86-91.

21 Y. Yang and W. M. Urban, Chem. Soc. Rev., 2013, 42, 7446-7467.

22 Y. Zhu, H. Xuan, J. Ren and L. Ge, Soft Matter, 2015, 11, 8452-8459.

23 I. Lagzi and D. Ueyama, Chem. Phys. Lett., 2009, 468, 188-192.

24 I. Lagzi, Langmuir, 2012, 28, 3350-3354.

25 W. Dyck, Can. J. Chem., 1971, 49, 1575-1578.

26 A. Pluen, P. A. Netti, R. K. Jain and D. A. Berk, Biophys. J., 1999, 77, 542-552.

27 M. Golmohamadi and K. J. Wilkinson, Carbohydr. Polym., 2013, 94, 82-87.

28 C. Mattisson, P. Roger, B. Jönsson, A. Axelsson and G. Zacchi, J. Chromatogr. B: Biomed. Sci. Appl., 2000, 743, 151-167.

29 S. Liang, J. Xu, L. Weng, H. Dai, X. Zhang and L. Zhang, J. Controlled Release, 2006, 115, 189-196.

30 P. Lundberg and P. W. Kuchel, Magn. Reson. Med., 1997, 37, 44-52.

31 E. O. Stejskal and J. E. Tanner, J. Chem. Phys., 1965, 42, 288-292.

32 C. S. Johnson, Jr., Prog. Nucl. Magn. Reson. Spectrosc., 1999, 34, 203-256.

33 H. Harned and D. French, Ann. N. Y. Acad. Sci., 1945, 44, 267-281.

34 H. Harned and R. Nuttall, J. Am. Chem. Soc., 1947, 69, 736-740.

35 H. Harned and R. Nuttall, J. Am. Chem. Soc., 1949, 71, 1460-1463.

36 H. Harned and C. Blake, J. Am. Chem. Soc., 1950, 72, 2265-2266.

37 H. Harned and C. H. Jr, J. Am. Chem. Soc., 1951, 73, 650-652.

38 H. Harned and R. Hudson, J. Am. Chem. Soc., 1951, 73, 3781-3783.

39 Z. Virányi, Á. Tóth and D. Horváth, J. Eng. Math., 2007, 59, 229-238.

40 Z. Virányi, Á. Tóth and D. Horváth, Phys. Rev. Lett., 2008, 100, 088301.

41 F. Bordi, C. Cametti and G. Paradossi, J. Phys. Chem., 1991, 95, 4883-4889. 